Document downloaded from:

http://hdl.handle.net/10251/84991

This paper must be cited as:

Hernández, AE.; Lattanzi, MB.; Thome Coppo, NJ. (2016). On some new pre-orders defined by weighted Drazin inverses. Applied Mathematics and Computation. 282:108-116. doi:10.1016/j.amc.2016.01.055.

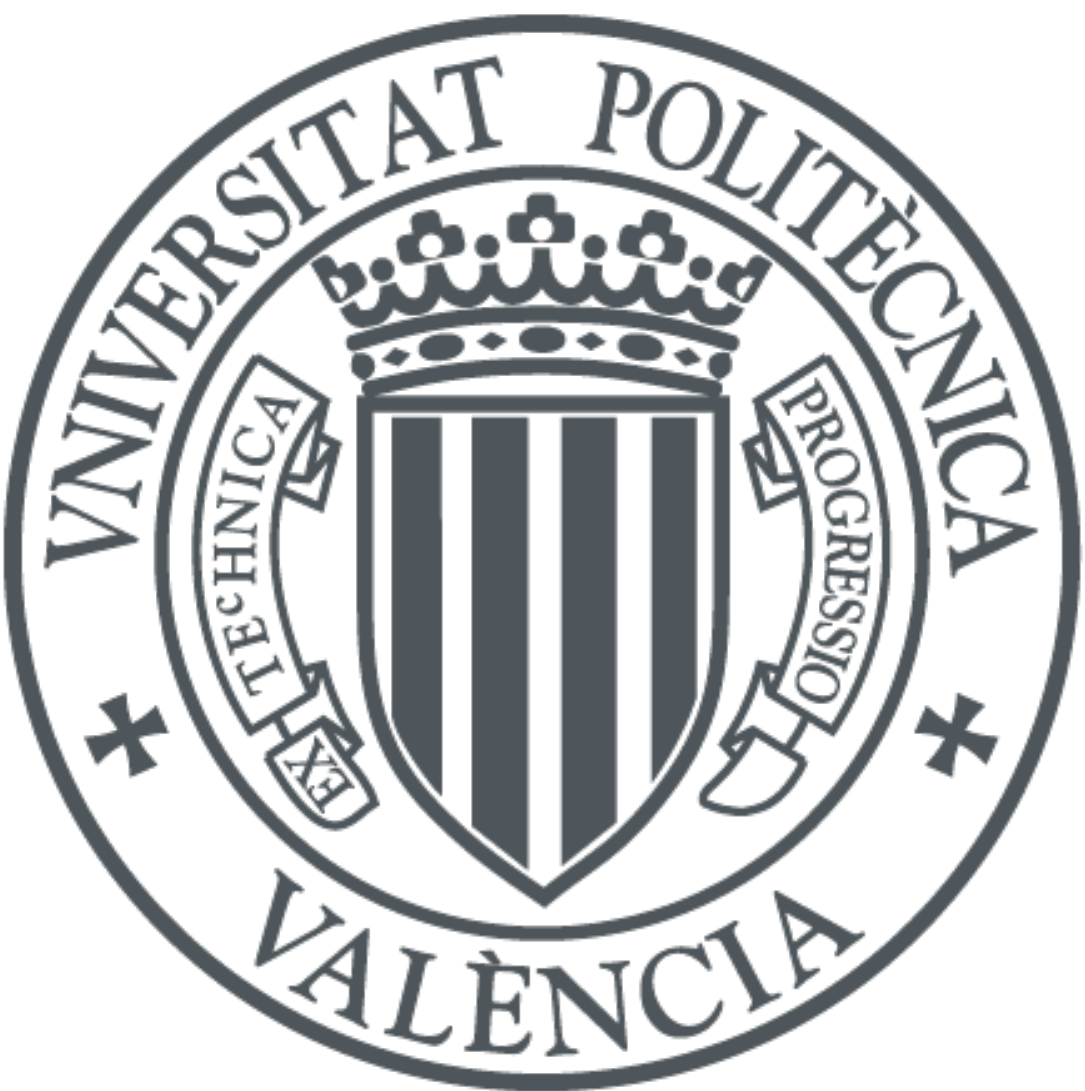

The final publication is available at

http://doi.org/10.1016/j.amc.2016.01.055

Copyright Elsevier

Additional Information 


\title{
On some new pre-orders defined by weighted Drazin inverses *
}
A. Hernández ${ }^{\dagger \ddagger}$
M. Lattanzi
N. Thome $e^{\S}$

\begin{abstract}
In this paper, we investigate new binary relations defined on the set of rectangular complex matrices based on the weighted Drazin inverse and give some characterizations of them. These relations become pre-orders and improve the results found by the authors in [Applied Mathematics and Computation, 219, 14 (2013), 73107318 ] as well as extend those known for square matrices. On the other hand, some new weighted partial orders are also defined and characterized. The advantages of these new relations compared to the ones considered in the mentioned paper are also pointed out.
\end{abstract}

AMS Classification: 15A09, 06A06

Keywords: Weighted Drazin inverse; Drazin pre-order; sharp partial order; minus partial order.

*This paper was partially supported by Universidad Nacional de La Pampa, Facultad de Ingeniería (grant Resol. No $155 / 14$ ).

${ }^{\dagger}$ Facultad de Ingeniería, Universidad Nacional de La Pampa, Calle 110 No 390, General Pico, La Pampa. Argentina. E-mail: aracelih@ing.unlpam.edu.ar.

${ }^{\ddagger}$ Facultad de Ciencias Exactas y Naturales, Universidad Nacional de La Pampa, Av. Uruguay 151, Santa Rosa, La Pampa. Argentina. E-mail: mblatt@exactas.unlpam.edu.ar .

§Instituto Universitario de Matemática Multidisciplinar, Universitat Politècnica de València, 46022, València, Spain. E-mail: njthome@mat.upv.es. This author was partially supported by Ministerio de Economía y Competitividad of Spain (grant DGI MTM2013-43678-P). 


\section{Introduction and background}

The main aim of this paper is to define and investigate some new pre-orders and some new partial orders on rectangular matrices by means of weighted Drazin inverses. On the one hand, the novelty of these relations is that they take into account the nilpotent parts of appropriate matrices of an arbitrary index. On the other hand, it is immediately seen that if we compare them to those recently studied in [9], defined only in terms of Drazin inverses, results are clearly improved obtaining some unexpected interesting features.

Whereas the relations that use a weight on both sides are shown that coincide in both papers, those including the weight on only one-side will extend properly the corresponding ones defined in [9] and will be different. An advantage derived from the present paper is that we can reduce from four to two the conditions that have to be verified for the comparison $A \preceq^{d, W} B$ to be true ([9, Theorem 2.3]). Another profitable consequence is that we can also reduce the quantity of Drazin inverses to be computed from two to only one.

Additionally, in this paper some new weighted partial orders are also defined and studied for rectangular matrices, again considering the nilpotent parts of appropriate matrices of an arbitrary index. These results are as well a generalization of those given, for matrices of index 1 , in [9, Section 5].

Let $\mathbb{C}^{m \times n}$ be the set of $m \times n$ complex matrices. For a given $A \in \mathbb{C}^{m \times n}$, the symbols $A^{*}, A^{-1}$ and $\mathcal{R}(A)$ denote the conjugate transpose, the inverse $(m=n)$ and the range of $A$. As usual, $I_{n}$ and $O_{n}$ denote the $n \times n$ identity and zero matrices, respectively. The subscripts will be omitted when no confusion is caused. For two given matrices $A \in \mathbb{C}^{t \times t}$ and $B \in \mathbb{C}^{(m-t) \times(n-t)}$ we denote by $A \oplus B$ the $m \times n$ matrix where $A$ is located in the $\mathrm{N}-\mathrm{W}$ corner, $B$ is in the $\mathrm{S}-\mathrm{E}$ corner and the other two blocks correspond to rectangular zero matrices of adequate sizes.

Let $A \in \mathbb{C}^{n \times n}$. The index of $A$, denoted by $\operatorname{ind}(A)$, is the smallest nonnegative integer $k$ such that $A^{k}$ and $A^{k+1}$ have the same rank. The only matrix $X \in \mathbb{C}^{n \times n}$ satisfying $X A X=X, A X=X A$ and $A^{k+1} X=A^{k}$, with $k=i n d(A)$, is called the Drazin inverse of $A$. The Drazin inverse of $A$ always exists and is denoted by $X=A^{D}$. It is clear that $A^{r+1} A^{D}=A^{r}$, for every integer $r \geq \operatorname{ind}(A)$ and $A^{r+1} A^{D}=A^{D} A^{r+1}$, for every integer $r \geq 0$. The group inverse of $A \in \mathbb{C}^{n \times n}$ is the unique matrix $A^{\#}$ that satisfies 
$A A^{\#} A=A, A^{\#} A A^{\#}=A^{\#}$ and $A A^{\#}=A^{\#} A$. If $A$ has index at most 1 , then $A^{D}=A^{\#}$. The Drazin inverse of a matrix can be computed by using a canonical representation for $A$. Indeed, for a given matrix $A \in \mathbb{C}^{n \times n}$ with $\operatorname{ind}(A)=k$ and $\operatorname{rank}\left(A^{k}\right)=a$, there are nonsingular matrices $P \in \mathbb{C}^{n \times n}$ and $C \in \mathbb{C}^{a \times a}$ such that $A=P(C \oplus N) P^{-1}$, where $N \in \mathbb{C}^{(n-a) \times(n-a)}$ is nilpotent with index $k$. Notice that $N$ is absent whether $k=0$, $N=O_{n-a}$ whether $k=1$ and $C$ is absent whether $a=0$. Along the entire paper only nontrivial cases will be considered. If $A$ is in the specified form then $A^{D}=P\left(C^{-1} \oplus O\right) P^{-1}$. Subsequently, we can write $A$ in the called core-nilpotent decomposition as $A=A_{1}+A_{2}$ where $A_{1}=P(C \oplus O) P^{-1}$ and $A_{2}=P(O \oplus N) P^{-1}$ for $A_{1}, A_{2}$ being the only matrices in these conditions (see [4]).

Recall that a binary relation on a set $\mathcal{S}$ which is reflexive and transitive is called a pre-order on $\mathcal{S}$. A partial order on $\mathcal{S}$ is a pre-order that also satisfies the antisymmetric property. The following relations are well known [10]. The minus partial order on $\mathbb{C}^{m \times n}$, denoted by $\leq^{-}$, is defined by $A \leq^{-} B$ if and only if $\operatorname{rank}(B-A)=\operatorname{rank}(B)-\operatorname{rank}(A)$, for $A, B \in \mathbb{C}^{m \times n}$. If $A, B \in \mathbb{C}^{n \times n}$ are two matrices with index at most 1 , it is said that $A$ is below $B$ under the sharp partial order if $A^{\#} A=A^{\#} B$ and $A A^{\#}=B A^{\#}$ and is denoted by $A \leq \# B$. Suppose that $A, B \in \mathbb{C}^{n \times n}$ are written in their respective core-nilpotent decompositions as $A=A_{1}+A_{2}$ and $B=B_{1}+B_{2}$. It is said that $A \preceq^{d} B$ if $A_{1} \leq^{\#} B_{1}$. The relation $\preceq^{d}$ is known as the Drazin pre-order. Observe that $A \preceq^{d} B$ is equivalent to $A^{D} A=A^{D} B$ and $A A^{D}=B A^{D}$.

Throughout this paper, a nonzero matrix $W \in \mathbb{C}^{n \times m}$ will be fixed to be used as a weight. The following definition was introduced by R.E. Cline and T.N.E. Greville in [6].

Definition 1.1 Let $W \in \mathbb{C}^{n \times m}$ be a nonzero matrix and $A \in \mathbb{C}^{m \times n}$. A matrix $X \in \mathbb{C}^{m \times n}$ is a $W$-weighted Drazin inverse of $A$ if the following conditions hold:

(a) $A W X=X W A$,

(b) $X=X W A W X$,

(c) $(A W)^{k+1} X W=(A W)^{k}$, for some integer $k \geq 0$.

The smallest integer $k$ satisfying condition (c) is denoted by $k_{1}=\operatorname{ind}(A W)$. 
The matrix $X$ in Definition 1.1 always exists, is unique and will be denoted by $X=$ $A^{D, W}$. Moreover, the equalities

$$
A^{D, W}=A\left((W A)^{D}\right)^{2}=\left((A W)^{D}\right)^{2} A, A^{D, W} W=(A W)^{D} \text { and } W A^{D, W}=(W A)^{D}
$$

hold (see [6]).

Notice that if $W \in \mathbb{C}^{n \times m}$ is a nonzero matrix and $A, X \in \mathbb{C}^{m \times n}$ are matrices satisfying item (a) in Definition 1.1, then it is easy to see that the following conditions are equivalent:

(b) $X=X W A W X$,

$\left(\mathrm{b}^{\prime}\right)(X W)^{2} A=X$,

$\left(\mathrm{b}^{\prime \prime}\right) A(W X)^{2}=X$.

Thus, condition (b) in Defintion 1.1 can be replaced with $\left(\mathrm{b}^{\prime}\right)$ or $\left(\mathrm{b}^{\prime \prime}\right)$.

Since $W=O$ yields to the trivial case $A^{D, W}=O$, this possibility is discarded. The next result will be used in what follows.

Theorem 1.1 [15, Theorem 2] If $W \in \mathbb{C}^{n \times m}$ is a nonzero matrix, $A \in \mathbb{C}^{m \times n}, k_{1}=$ $\operatorname{ind}(A W)$ and $k_{2}=\operatorname{ind}(W A)$ then there exist four nonsingular matrices $P \in \mathbb{C}^{m \times m}$, $Q \in \mathbb{C}^{n \times n}, A_{1}, W_{1} \in \mathbb{C}^{t \times t}$ and two matrices $A_{2} \in \mathbb{C}^{(m-t) \times(n-t)}$ and $W_{2} \in \mathbb{C}^{(n-t) \times(m-t)}$ such that $A_{2} W_{2}$ and $W_{2} A_{2}$ are nilpotent of indices $k_{1}$ and $k_{2}$, respectively, with

$$
A=P\left(A_{1} \oplus A_{2}\right) Q^{-1} \quad \text { and } \quad W=Q\left(W_{1} \oplus W_{2}\right) P^{-1} .
$$

In this case,

$$
A^{D, W}=P\left(\left(W_{1} A_{1} W_{1}\right)^{-1} \oplus O\right) Q^{-1} .
$$

Observe that Theorem 1.1 can also be established for $k=\max \left\{k_{1}, k_{2}\right\}$ (see [14]). Moreover, the matrix $W$ can be seen as a weight needed to transform the rectangular matrix $A$ into two square ones, namely, $A W$ and $W A$.

For a most extensive study on generalized inverses and matrix partial orders and pre-orders the authors refer the reader to $[1,2,3,4,7,8,11,12,13]$.

This paper is organized as follows. Section 2 introduces and characterizes the binary relation $\preceq^{D, W}$ on rectangular matrices. In order to provide a pre-order on rectangular 
complex matrices based on the weighted Drazin inverse, the new binary relation $\preceq^{D, W}$ is defined and characterized on rectangular matrices in Section 2. This relation requires only 2 equalities, as well as the majority of the well-known orders or pre-orders defined on the basis of a generalized inverse, as for example star order, sharp order, Drazin pre-order, minus order, etc. This relation becomes equivalent to $\preceq^{d, W}$ (defined in [9] in terms of the Drazin inverse), and this new characterization simplifies considerably the known one. Additionally, several computational advantages of the new relation are remarked. Section 3 presents the one-sided relations $\preceq^{D, W, r}$ and $\preceq^{D, W, \ell}$ (not considered in [9]) corresponding to $\preceq^{D, W}$ and their characterizations. It is shown that they are pre-orders and some relationship with the pre-orders $\preceq^{d, W, r}$ and $\preceq^{d, W, \ell}$ introduced in [9] are given. It is proved that $\preceq^{d, W, r} \subseteq \preceq^{D, W, r}$ and $\preceq^{d, W, \ell} \subseteq \preceq^{D, W, \ell}$ and that the opposite inclusions do not hold. In Section 4, new weighted partial orders on rectangular matrices are introduce and characterized.

\section{A pre-order defined by the weighted Drazin inverse}

The Drazin pre-order can be defined on square matrices thanks to the fact that Drazin inverses always exist for square matrices. Since it is not possible to extend this preorder directly to rectangular matrices, we will take advantage from the weighted Drazin inverses that always exist for rectangular matrices. Then the idea is to consider some binary relations on the set of rectangular matrices by using weighted Drazin inverses.

Definition 2.1 Let $W \in \mathbb{C}^{n \times m}$ be a nonzero matrix and $A, B \in \mathbb{C}^{m \times n}$. It is said that $A \preceq^{D, W} B$ if $(A W) A^{D, W}=(B W) A^{D, W}$ and $A^{D, W}(W A)=A^{D, W}(W B)$.

It is remarkable that only one Drazin inverse has to be computed in the previous Definition because of the expressions for $A^{D, W}$ given in (1).

Theorem 2.1 Let $W \in \mathbb{C}^{n \times m}$ be a nonzero matrix and $A, B \in \mathbb{C}^{m \times n}$. If $A$ and $W$ are written as in (2), then the following conditions are equivalent:

(a) $A \preceq^{D, W} B$,

(b) there exists $B_{2} \in \mathbb{C}^{(m-t) \times(n-t)}$ such that $B=P\left(A_{1} \oplus B_{2}\right) Q^{-1}$. 
Proof. Consider the following partition of $B$

$$
B=P\left[\begin{array}{ll}
B_{1} & B_{3} \\
B_{4} & B_{2}
\end{array}\right] Q^{-1},
$$

according to the size of blocks in $A$. From (2) and (3) we get $A^{D, W}(W A)=P\left(W_{1}^{-1} \oplus\right.$ $O) Q^{-1}$ and

$$
A^{D, W}(W B)=P\left[\begin{array}{cc}
W_{1}^{-1} A_{1}^{-1} B_{1} & W_{1}^{-1} A_{1}^{-1} B_{3} \\
O & O
\end{array}\right] Q^{-1} .
$$

Since $A^{D, W}(W A)=A^{D, W}(W B)$, it then results $B_{1}=A_{1}$ and $B_{3}=O$. Similarly, the equality $(A W) A^{D, W}=(B W) A^{D, W}$ leads to $B_{4}=O$. Hence, (a) $\Longrightarrow(\mathrm{b})$ holds. The converse is straightforward.

Remark 2.1 From Theorem 2.1 it is clear that $\preceq^{D, W}$ does not preserve the rank function. Indeed, for a fixed matrix $A$ decomposed as in (2) with $A_{2}$ being neither nonzero nor a full rank matrix, if we choose $B_{2}$ as the zero matrix, as $A_{2}$ or as a full rank matrix we get that $\operatorname{rank}(B)$ is respectively less, equal or greater than $\operatorname{rank}(A)$.

Remark 2.2 In general, the index of (the square matrices corresponding to) two matrices related under $\preceq^{D, W}$ is not linked. That is, if $A \preceq^{D, W} B$ with $\operatorname{ind}(A W)=k_{1}$ and $\operatorname{ind}(B W)=k_{2}$ we know that each one of the possibilities $k_{2}<k_{1}, k_{2}=k_{1}$ or $k_{2}>k_{1}$ may occur as the following example shows. For the matrices

$$
\begin{gathered}
A=\left[\begin{array}{llll}
2 & 0 & 0 & 0 \\
0 & 1 & 0 & 0 \\
0 & 0 & 0 & 1
\end{array}\right], \quad W=\left[\begin{array}{lll}
1 & 0 & 0 \\
0 & 1 & 0 \\
0 & 0 & 1 \\
0 & 0 & 0
\end{array}\right] . \\
B_{1}=\left[\begin{array}{llll}
2 & 0 & 0 & 0 \\
0 & 1 & 0 & 0 \\
0 & 0 & 5 & 0
\end{array}\right], \quad B_{2}=\left[\begin{array}{llll}
2 & 0 & 0 & 0 \\
0 & 1 & 0 & 0 \\
0 & 0 & 0 & 5
\end{array}\right], \quad B_{3}=\left[\begin{array}{llll}
2 & 0 & 0 & 0 \\
0 & 0 & 1 & 0 \\
0 & 0 & 0 & 0
\end{array}\right]
\end{gathered}
$$


it can be easily proved that $A \preceq^{D, W} B_{1}, A \preceq^{D, W} B_{2}, A \preceq^{D, W} B_{3}$, ind $(A W)=k_{1}=1$, $\operatorname{ind}\left(B_{1} W\right)=0, \operatorname{ind}\left(B_{2} W\right)=1$ and $\operatorname{ind}\left(B_{3} W\right)=2$. Of course, it similarly occurs with the left counterpart $W A$ and $W B$.

Notice that proof of Theorem 2.1 only uses the nonsingularity of $A_{1}$ and $W_{1}$ that Theorem 1.1 states, but not the remaining properties about nilpotence.

Theorem 2.2 Let $W \in \mathbb{C}^{n \times m}$ be a nonzero matrix. The binary relation $\preceq^{D, W}$ defined on $\mathbb{C}^{m \times n}$ is a pre-order.

Proof. By Definition 2.1 it is immediate that $\preceq^{D, W}$ is reflexive. Now, suppose that $A, B, C \in \mathbb{C}^{m \times n}$ satisfy $A \preceq^{D, W} B$ and $B \preceq^{D, W} C$. Since $A \preceq^{D, W} B$, Theorem 2.1 assures the existence of nonsingular matrices $P \in \mathbb{C}^{m \times m}, Q \in \mathbb{C}^{n \times n}, A_{1}, W_{1} \in \mathbb{C}^{t \times t}$ and matrices $B_{2} \in \mathbb{C}^{(m-t) \times(n-t)}$ and $W_{2} \in \mathbb{C}^{(n-t) \times(m-t)}$ such that $A=P\left(A_{1} \oplus A_{2}\right) Q^{-1}$, $W=Q\left(W_{1} \oplus W_{2}\right) P^{-1}$ and $B=P\left(A_{1} \oplus B_{2}\right) Q^{-1}$. Thus,

$$
(W B)^{D}=Q\left(W_{1} A_{1} \oplus W_{2} B_{2}\right)^{D} Q^{-1}=Q\left(\left(W_{1} A_{1}\right)^{-1} \oplus\left(W_{2} B_{2}\right)^{D}\right) Q^{-1}
$$

which implies $\left[(W B)^{D}\right]^{2}=Q\left(\left(W_{1} A_{1}\right)^{-2} \oplus\left[\left(W_{2} B_{2}\right)^{D}\right]^{2}\right) Q^{-1}$. By $(1), B^{D, W}=B\left[(W B)^{D}\right]^{2}=$ $P\left(A_{1}\left(W_{1} A_{1}\right)^{-2} \oplus B_{2}\left[\left(W_{2} B_{2}\right)^{D}\right]^{2}\right) Q^{-1}=P\left(A_{1}^{-1} W_{1}^{-2} \oplus B_{2}^{D, W_{2}}\right) Q^{-1}$ (even for $\left.W_{2}=O\right)$. Now, from $B \preceq^{D, W} C$ we have $(B W) B^{D, W}=(C W) B^{D, W}$ and $B^{D, W}(W B)=B^{D, W}(W C)$. Note that Theorem 2.1 can be applied to $B \preceq^{D, W} C$ due to the nonsingularity of the matrices $A_{1}$ and $W_{1}$ involved in the decompositions of $B$ and $W$. Thus, $C=P\left(A_{1} \oplus C_{2}\right) Q^{-1}$, for some $C_{2} \in \mathbb{C}^{(m-t) \times(n-t)}$. Now, using that $\left.A^{D, W}=A\left[(W A)^{D}\right)\right]^{2}=P\left(A_{1}^{-1} W_{1}^{-2} \oplus O\right) Q^{-1}$, it is easy to see that $A^{D, W}(W A)=A^{D, W}(W C)$ and $(A W) A^{D, W}=(C W) A^{D, W}$. Hence, $A \preceq^{D, W} C$ and thus transitivity holds.

In general, the relation $\preceq^{D, W}$ is not antisymmetric as the following example shows.

Example 2.1 Consider the matrices $A, B_{1}$ and $W$ given in Remark 2.2. It is clear that $A$ and $W$ are written as in Theorem 1.1, hence $A \preceq^{D, W} B_{1}$ by Theorem 2.1. Similarly, $B_{1} \preceq^{D, W} A$, but $A \neq B_{1}$.

The next result will be used in what follows. 
Lemma 2.1 Let $W \in \mathbb{C}^{n \times m}$ be a nonzero matrix, $A, B \in \mathbb{C}^{m \times n}, k_{1}=\operatorname{ind}(A W)$ and $k_{2}=\operatorname{ind}(W A)$. Then

(a) $(A W)(A W)^{D}=(B W)(A W)^{D}$ if and only if $(A W)^{k_{1}+1}=(B W)(A W)^{k_{1}}$,

(b) $(W A)^{D}(W A)=(W A)^{D}(W B)$ if and only if $(W A)^{k_{2}+1}=(W A)^{k_{2}}(W B)$.

Proof. It is enough to prove item (a) because item (b) follows similarly.

If we suppose that $(A W)(A W)^{D}=(B W)(A W)^{D}$ holds, clearly the equality

$$
(A W)(A W)^{D}(A W)^{k_{1}+1}=(B W)(A W)^{D}(A W)^{k_{1}+1}
$$

also holds and Drazin inverse definition yields $(A W)^{k_{1}+1}=(B W)(A W)^{k_{1}}$, as we desired. The converse will be proved by induction on $k_{1}$. The $k_{1}=0$ case holds vacuously. Suppose that $(A W)^{k_{1}+1}=(B W)(A W)^{k_{1}}$ implies $(A W)(A W)^{D}=(B W)(A W)^{D}$ for same integer $k_{1} \geq 0$ and that $(A W)^{\left(k_{1}+1\right)+1}=(B W)(A W)^{k_{1}+1}$ is as well satisfied. Then

$$
(A W)^{k_{1}+1}(A W)(A W)^{D}=(B W)(A W)^{k_{1}}(A W)(A W)^{D}
$$

So, by Drazin inverse definition we arrive at $(A W)^{k_{1}+1}=(B W)(A W)^{k_{1}}$. Hence, by induction hypothesis we have $(A W)(A W)^{D}=(B W)(A W)^{D}$.

We next characterize the relation $A \preceq^{D, W} B$ in terms of the weighted Drazin inverse of $A$, the Drazin inverse of $A W$ and $W A$, the index of $A W$ and $W A$ and also using adequate blocks of matrices $A, B$ and $W$.

Theorem 2.3 Let $W \in \mathbb{C}^{n \times m}$ be a nonzero matrix, $A, B \in \mathbb{C}^{m \times n}, k_{1}=$ ind $(A W)$ and $k_{2}=\operatorname{ind}(W A)$. The following conditions are equivalent:

(a) $A \preceq^{D, W} B$.

(b) $(A W)\left(A^{D, W} W\right)=(B W)\left(A^{D, W} W\right)$ and $\left(W A^{D, W}\right)(W A)=\left(W A^{D, W}\right)(W B)$.

(c) $(A W)(A W)^{D}=(B W)(A W)^{D}$ and $(W A)^{D}(W A)=(W A)^{D}(W B)$.

(d) $(A W)^{k_{1}+1}=(B W)(A W)^{k_{1}}$ and $(W A)^{k_{2}+1}=(W A)^{k_{2}}(W B)$.

(e) $(A W)^{k+1}=(B W)(A W)^{k}$ and $(W A)^{k+1}=(W A)^{k}(W B)$ for all integer $k \geq \max \left\{k_{1}, k_{2}\right\}$. 
(f) There exist nonsingular matrices $P, Q, A_{1}, W_{1}, B_{1}^{2}, W_{1}^{2}$, and matrices $A_{2}, B_{2}^{2}, W_{2}^{2}$, all of them of suitable sizes, such that

$$
A=P\left(A_{1} \oplus A_{2}\right) Q^{-1}, \quad B=P\left(A_{1} \oplus\left(B_{1}^{2} \oplus B_{2}^{2}\right)\right) Q^{-1}
$$

and $W=Q\left(W_{1} \oplus\left(W_{1}^{2} \oplus W_{2}^{2}\right)\right) P^{-1}$, where $A_{2}\left(W_{1}^{2} \oplus W_{2}^{2}\right),\left(W_{1}^{2} \oplus W_{2}^{2}\right) A_{2}, B_{2}^{2} W_{2}^{2}$ and $W_{2}^{2} B_{2}^{2}$ are nilpotent matrices.

Proof. (a) $\Longrightarrow$ (b) It is immediate from Definition 2.1.

(b) $\Longrightarrow$ (c) It follows from (1).

(c) $\Longrightarrow$ (a) Post-multiplying $(A W)(A W)^{D}=(B W)(A W)^{D}$ by $(A W)^{D} A$ it then follows $(A W)\left((A W)^{D}\right)^{2} A=(B W)\left((A W)^{D}\right)^{2} A$. By $(1)$, the equality $(A W) A^{D, W}=(B W) A^{D, W}$ holds. The other equality follows similarly.

(c) $\Longleftrightarrow$ (d) It is immediate from Lemma 2.1.

(d) $\Longrightarrow(\mathrm{e})$ Let $k$ be an integer such that $k \geq m$ where $m=\max \left\{k_{1}, k_{2}\right\}$. If $m=k_{1}$, from $(A W)^{k_{1}+1}=(B W)(A W)^{k_{1}}$ we have

$$
(A W)^{k+1}=(A W)^{k_{1}+1}(A W)^{k-m}=(B W)(A W)^{k_{1}}(A W)^{k-m}=(B W)(A W)^{k} .
$$

We can proceed analogously to obtain the $m=k_{2}$ case.

$(\mathrm{e}) \Longrightarrow(\mathrm{d})$ Let $k \geq \max \left\{k_{1}, k_{2}\right\}$ be an integer such that (i) $(A W)^{k+1}=(B W)(A W)^{k}$ and (ii) $(W A)^{k+1}=(W A)^{k}(W B)$ are satisfied.

Denote by $m_{1}$ the smallest integer $k \geq \max \left\{k_{1}, k_{2}\right\}$ for which (i) holds. So, $m_{1} \geq k_{1}$ satisfies $(A W)^{m_{1}+1}=(B W)(A W)^{m_{1}}$. Set $t=m_{1}-k_{1}$. Clearly, $t \geq 0$. Assume that $t \geq 1$ because the $t=0$ case is trivial. Multiplying $(A W)^{k_{1}+t+1}=(B W)(A W)^{k_{1}+t}$ by $\left((A W)^{D}\right)^{t}$ on the right side and using that $\operatorname{ind}(A W)=k_{1}$ we get $(A W)^{k_{1}+1}=(B W)(A W)^{k_{1}}$.

On the other hand, if we denote by $m_{2}$ the smallest integer $k \geq \max \left\{k_{1}, k_{2}\right\}$ that satisfies (ii) and we set $s=m_{2}-k_{2}$, it can be similarly seen that $(A W)^{k_{2}+s+1}=(B W)(A W)^{k_{2}+s}$ implies $(A W)^{k_{2}+1}=(B W)(A W)^{k_{2}}$.

(c) $\Longrightarrow$ (f) Suppose that $A, B \in \mathbb{C}^{m \times n}$ satisfy (c). By Theorem 1.1 there are nonsingular matrices $P_{A} \in \mathbb{C}^{m \times m}, Q_{A} \in \mathbb{C}^{n \times n}, A_{1}, W_{1} \in \mathbb{C}^{t_{A} \times t_{A}}$, and matrices $A_{2}^{\prime} \in \mathbb{C}^{\left(m-t_{A}\right) \times\left(n-t_{A}\right)}$, $W_{2} \in \mathbb{C}^{\left(n-t_{A}\right) \times\left(m-t_{A}\right)}$ such that

$$
A=P_{A}\left(A_{1} \oplus A_{2}^{\prime}\right) Q_{A}^{-1} \quad \text { and } \quad W=Q_{A}\left(W_{1} \oplus W_{2}\right) P_{A}^{-1}
$$


where $A_{2}^{\prime} W_{2}$ and $W_{2} A_{2}^{\prime}$ are nilpotent matrices of indices $k_{1}=\operatorname{ind}(A W)$ and $k_{2}=$ ind $(W A)$, respectively. Moreover, $A^{D, W}=P_{A}\left(\left(W_{1} A_{1} W_{1}\right)^{-1} \oplus O\right) Q_{A}^{-1}$. Hence, by (1) $A^{D, W} W=(A W)^{D}=P_{A}\left(\left(A_{1} W_{1}\right)^{-1} \oplus O\right) P_{A}^{-1}$ and $W A^{D, W}=(W A)^{D}=Q_{A}\left(\left(W_{1} A_{1}\right)^{-1} \oplus\right.$ O) $Q_{A}^{-1}$.

Let us consider the following partition of $B$

$$
B=P_{A}\left[\begin{array}{cc}
B_{1} & B_{3} \\
B_{4} & B_{2}
\end{array}\right] Q_{A}^{-1},
$$

according to the size of the blocks of $A$. From $(A W)(A W)^{D}=(B W)(A W)^{D}$, after making same calculations it is obtained $B_{1}=A_{1}$ and $B_{4}=O$. Now, from equality $(W A)^{D}(W A)=(W A)^{D}(W B)$, it results $B_{3}=O$, i.e., $B=P_{A}\left(A_{1} \oplus B_{2}\right) Q_{A}^{-1}$.

Suppose that $W_{2} \neq O$. Applying Theorem 1.1 to matrices $B_{2} \in \mathbb{C}^{\left(m-t_{A}\right) \times\left(n-t_{A}\right)}$ and $W_{2} \in \mathbb{C}^{\left(n-t_{A}\right) \times\left(m-t_{A}\right)}$, there exist nonsingular matrices $R \in \mathbb{C}^{\left(m-t_{A}\right) \times\left(m-t_{A}\right)}, S \in$ $\mathbb{C}^{\left(n-t_{A}\right) \times\left(n-t_{A}\right)}, B_{1}^{2}, W_{1}^{2} \in \mathbb{C}^{t \times t}$, and matrices $B_{2}^{2} \in \mathbb{C}^{\left(m-t_{A}-t\right) \times\left(n-t_{A}-t\right)}, W_{2}^{2} \in \mathbb{C}^{\left(n-t_{A}-t\right) \times\left(m-t_{A}-t\right)}$ satisfying

$$
B_{2}=R\left(B_{1}^{2} \oplus B_{2}^{2}\right) S^{-1} \quad \text { and } \quad W_{2}=S\left(W_{1}^{2} \oplus W_{2}^{2}\right) R^{-1}
$$

where $B_{2}^{2} W_{2}^{2}$ and $W_{2}^{2} B_{2}^{2}$ are nilpotent.

Consider the matrices $P \in \mathbb{C}^{m \times m}$ and $Q \in \mathbb{C}^{n \times n}$ defined by

$$
P=P_{A}\left(I_{t_{A}} \oplus R\right) \quad \text { and } \quad Q=Q_{A}\left(I_{t_{A}} \oplus S\right) .
$$

Replacing (4) and (5) in the expressions of $A, B$ and $W$ and setting $A_{2}=R^{-1} A_{2}^{\prime} S$ we arrive at

$$
A=P\left(A_{1} \oplus A_{2}\right) Q^{-1}, \quad B=P\left(A_{1} \oplus R^{-1} B_{2} S\right) Q^{-1}, \quad W=Q\left(W_{1} \oplus S^{-1} W_{2} R\right) P^{-1}
$$

We notice that the $W_{2}=O$ case can be also written as in (f) with $W_{1}^{2}=O$ and $W_{2}^{2}=O$.

(f) $\Longrightarrow$ (c) It is straightforward.

Now, we establish some relationships between known pre-orders. As a consequence of Theorem 2.3 we can compare the pre-order $\preceq^{D, W}$ to the one studied in [9], that is, $A \preceq^{d, W} B$ if and only if $A W \preceq^{d} B W$ and $W A \preceq^{d} W B$. For more details, we refer the reader to $[9$, Theorem 2.3$]$. 
Proposition 2.1 Let $W \in \mathbb{C}^{n \times m}$ be a nonzero matrix, $A, B \in \mathbb{C}^{m \times n}$, ind $(A W)=k_{1}$ and $\operatorname{ind}(W A)=k_{2}$. Then

(a) $A \preceq^{D, W} B$ if and only if $A \preceq^{d, W} B$.

(b) $(W A)^{D}(W A)=(W A)^{D}(W B)$ and $(A W)(A W)^{D}=(B W)(A W)^{D}$ if and only if $(A W)^{D}(A W)=(A W)^{D}(B W)=(B W)(A W)^{D}$ and $(W A)^{D}(W A)=(W A)^{D}(W B)=$ $(W B)(W A)^{D}$.

(c) $(B W)(A W)^{k_{1}}=(A W)^{k_{1}+1}$ and $(W A)^{k_{2}}(W B)=(W A)^{k_{2}+1}$ if and only if $(A W)^{k_{1}+1}=$ $(A W)^{k_{1}}(B W)=(B W)(A W)^{k_{1}}$ and $(W A)^{k_{2}+1}=(W A)^{k_{2}}(W B)=(W B)(W A)^{k_{2}}$.

Remark 2.3 An important remark is that we have reduced from four ([9, Theorem 2.3]) to two (Proposition 2.1) the conditions to be verified for the relation $A \preceq^{d, W} B$ to be true. Even more, in order to assure that $A \preceq^{d, W} B$, we have now to compute only one Drazin inverse (namely $(A W)^{D}$ or $(W A)^{D}$ in the expression for $A^{D, W}$ ) while in ([9, Theorem 2.3]) we must compute two.

\section{One-sided pre-orders}

In this section we consider the right and left-sided relations associated to $\preceq^{D, W}$ and compare them to the relations $\preceq^{d, W, r}$ and $\preceq^{d, W, \ell}$ defined in [9], respectively. Contrary to the intuition, in these cases they are not equivalent as we will show in what follows.

Definition 3.1 Let $W \in \mathbb{C}^{n \times m}$ be a nonzero matrix and $A, B \in \mathbb{C}^{m \times n}$. It is said that

(a) $A \preceq^{D, W, r} B$ if $(A W) A^{D, W}=(B W) A^{D, W}$.

(b) $A \preceq^{D, W, \ell} B$ if $A^{D, W}(W A)=A^{D, W}(W B)$.

From Definition 2.1 it is clear that $A \preceq^{D, W} B$ if and only if $A \preceq^{D, W, r} B$ and $A \preceq^{D, W, \ell}$ $B$.

Using (1) it is easy to see that $A \preceq^{D, W, r} B$ if and only if $(A W)(A W)^{D}=(B W)(A W)^{D}$. Similarly, $A \preceq^{D, W, \ell} B$ if and only if $(W A)^{D}(W A)=(W A)^{D}(W B)$. 
In what follows, we will show that the relation $\preceq^{D, W, r}$ is a pre-order. The fact that $\preceq^{D, W, \ell}$ is also a pre-order can be deduced from Theorem 3.1 and noticing that $A \preceq^{D, W, \ell} B$ if and only if $A^{*} \preceq^{D, W^{*}, r} B^{*}$.

Theorem 3.1 Let $W \in \mathbb{C}^{n \times m}$ be a nonzero matrix. The relation $\preceq^{D, W, r}$ defined on $\mathbb{C}^{m \times n}$ is a pre-order.

Proof. Clearly $\preceq^{D, W, r}$ is reflexive. We shall prove that it is transitive. Let $A, B, C \in$ $\mathbb{C}^{m \times n}$ satisfying $A \preceq^{D, W, r} B$ and $B \preceq^{D, W, r} C$. Suppose that $A$ and $W$ are written as in (2). Consider the following partitions of $B$ and $C$

$$
B=P\left[\begin{array}{ll}
B_{1} & B_{3} \\
B_{4} & B_{2}
\end{array}\right] Q^{-1}, \quad C=P\left[\begin{array}{ll}
C_{1} & C_{3} \\
C_{4} & C_{2}
\end{array}\right] Q^{-1},
$$

according to the size of blocks in $A$. Since $(A W)(A W)^{D}=(B W)(A W)^{D}$, it results $B_{1}=A_{1}$ and $B_{4}=O$. Hence,

$$
B=P\left[\begin{array}{cc}
A_{1} & B_{3} \\
O & B_{2}
\end{array}\right] Q^{-1} \quad \text { and } \quad B W=P\left[\begin{array}{cc}
A_{1} W_{1} & B_{3} W_{2} \\
O & B_{2} W_{2}
\end{array}\right] P^{-1} .
$$

Using the fact that for square matrices $M_{1}$ and $M_{2}$ and for a nonsingular matrix $P$, if $M_{2}=P M_{1} P^{-1}$ then $M_{2}^{D}=P M_{1}^{D} P^{-1}$, and from [4, Theorem 7.7.1], there exists a matrix $X$ of the adequate size such that

$$
(B W)^{D}=P\left[\begin{array}{cc}
\left(A_{1} W_{1}\right)^{-1} & X \\
O & \left(B_{2} W_{2}\right)^{D}
\end{array}\right] P^{-1} .
$$

Since $B \preceq^{D, W, r} C$, we have $(B W)(B W)^{D}=(C W)(B W)^{D}$. Making some calculations it is obtained that

$$
\begin{aligned}
& (B W)(B W)^{D}=P\left[\begin{array}{cc}
I & A_{1} W_{1} X+B_{3} W_{2}\left(B_{2} W_{2}\right)^{D} \\
O & B_{2} W_{2}\left(B_{2} W_{2}\right)^{D}
\end{array}\right] Q^{-1} \text { and } \\
& (C W)(B W)^{D}=P\left[\begin{array}{cc}
C_{1} A_{1}^{-1} & C_{1} W_{1} X+C_{3} W_{2}\left(B_{2} W_{2}\right)^{D} \\
C_{4} A_{1}^{-1} & C_{4} W_{1} X+C_{2} W_{2}\left(B_{2} W_{2}\right)^{D}
\end{array}\right] P^{-1},
\end{aligned}
$$

from where we have $C_{1}=A_{1}$ and $C_{4}=O$. Hence, it is easy to verify that $A \preceq^{D, W, r} C$; so $\preceq^{D, W, r}$ is transitive.

Analogously to Theorem 2.3 we can prove the following result. 
Theorem 3.2 Let $W \in \mathbb{C}^{n \times m}$ be a nonzero matrix, $A, B \in \mathbb{C}^{m \times n}$ and $k_{1}=\operatorname{ind}(A W)$.

The following conditions are equivalent:

(a) $A \preceq^{D, W, r} B$.

(b) $(A W)\left(A^{D, W} W\right)=(B W)\left(A^{D, W} W\right)$.

(c) $(A W)(A W)^{D}=(B W)(A W)^{D}$.

(d) $(A W)^{k_{1}+1}=(B W)(A W)^{k_{1}}$.

(e) $(A W)^{k+1}=(B W)(A W)^{k}$, for all integer $k \geq k_{1}$.

(f) There exist nonsingular matrices $P, Q, A_{1}, W_{1}, B_{1}^{2}, W_{1}^{2}$, and there exist $A_{2}, B_{3}, B_{2}^{2}$, $W_{2}^{2}$, all of them of suitable sizes, satisfying

$$
\begin{gathered}
A=P\left(A_{1} \oplus A_{2}\right) Q^{-1}, \quad B=P\left[\begin{array}{cc}
A_{1} & B_{3} \\
O & B_{1}^{2} \oplus B_{2}^{2}
\end{array}\right] Q^{-1}, \\
W=Q\left(W_{1} \oplus\left(W_{1}^{2} \oplus W_{2}^{2}\right)\right) P^{-1}
\end{gathered}
$$

where $A_{2}\left(W_{1}^{2} \oplus W_{2}^{2}\right),\left(W_{1}^{2} \oplus W_{2}^{2}\right) A_{2}, B_{2}^{2} W_{2}^{2}$ and $W_{2}^{2} B_{2}^{2}$ are nilpotent matrices.

We recall that the binary relation $\preceq^{d, W, r}$ was defined in [9] by $A \preceq^{d, W, r} B$ if $(A W)^{D}(A W)=$ $(A W)^{D}(B W)$ and $(A W)(A W)^{D}=(B W)(A W)^{D}$. It is clear that $\preceq^{d, W, r}$ is a subset of $\preceq^{D, W, r}$ but the converse is not true, as we show in the following example.

Example 3.1 Let us consider the matrices

$$
A=\left[\begin{array}{ll}
1 & 0 \\
0 & 0 \\
0 & 0
\end{array}\right], \quad B=\left[\begin{array}{cc}
1 & 1 \\
0 & 0 \\
0 & 0
\end{array}\right] \quad \text { and } \quad W=\left[\begin{array}{ccc}
2 & 0 & 0 \\
0 & 1 & 0
\end{array}\right] \text {. }
$$

It is easy to verify that $(A W)(A W)^{D}=(B W)(A W)^{D}=\left[\begin{array}{lll}1 & 0 & 0 \\ 0 & 0 & 0 \\ 0 & 0 & 0\end{array}\right]$, this is, $A \preceq \preceq^{D, W, r}$ B. However, $(A W)^{D}(A W) \neq(A W)^{D}(B W)$ because $(A W)^{D}(B W)=\left[\begin{array}{ccc}1 & \frac{1}{2} & 0 \\ 0 & 0 & 0 \\ 0 & 0 & 0\end{array}\right]$. Hence, $A \npreceq^{d, W, r} B$. 
Theorem 3.3 Let $W \in \mathbb{C}^{n \times m}$ be a nonzero matrix, $A, B \in \mathbb{C}^{m \times n}$ and $k_{2}=\operatorname{ind}(W A)$. The following conditions are equivalent:

(a) $A \preceq^{D, W, \ell} B$.

(b) $\left(W A^{D, W}\right)(W A)=\left(W A^{D, W}\right)(W B)$.

(c) $(W A)^{D}(W A)=(W A)^{D}(W B)$.

(d) $(W A)^{k_{2}+1}=(W A)^{k_{2}}(W B)$.

(e) $(W A)^{k+1}=(W A)^{k}(W B)$ for all integer $k \geq k_{2}$.

(f) There exist nonsingular matrices $P, Q, A_{1}, W_{1}, B_{1}^{2}, W_{1}^{2}$, and matrices $A_{2}, B_{4}, B_{2}^{2}$, $W_{2}^{2}$, all of them of suitable sizes, satisfying

$$
\begin{gathered}
A=P\left(A_{1} \oplus A_{2}\right) Q^{-1}, \quad B=P\left[\begin{array}{cc}
A_{1} & O \\
B_{4} & B_{1}^{2} \oplus B_{2}^{2}
\end{array}\right] Q^{-1}, \\
W=Q\left(W_{1} \oplus\left(W_{1}^{2} \oplus W_{2}^{2}\right)\right) P^{-1}
\end{gathered}
$$

where $A_{2}\left(W_{1}^{2} \oplus W_{2}^{2}\right),\left(W_{1}^{2} \oplus W_{2}^{2}\right) A_{2}, B_{2}^{2} W_{2}^{2}$ and $W_{2}^{2} B_{2}^{2}$ are nilpotent matrices.

Analogously, the binary relation $\preceq^{d, W, \ell}$ defined in [9] is a subset of $\preceq^{D, W, \ell}$, but the converse is not true, as it can be shown considering the conjugate transpose of matrices in Example 3.1.

Notice that similar observations to those in Remarks 2.1 and 2.2 can be also done for the relations $\preceq^{D, W, r}$ and $\varliminf^{D, W, \ell}$.

The last comment in Remark 2.3 is the reason why next equivalences are not included in Theorems 2.3, 3.2 and 3.3, that is, we can simplify even more the expressions.

Theorem 3.4 Let $W \in \mathbb{C}^{n \times m}$ be a nonzero matrix and $A, B \in \mathbb{C}^{m \times n}$. The following statements are valid:

(a) $A \preceq^{D, W, r} B$ if and only if $A(W A)^{D}=B(W A)^{D}$ if and only if $(A W)^{D} A=B(W A)^{D}$.

(b) $A \preceq^{D, W, \ell} B$ if and only if $(A W)^{D} A=(A W)^{D} B$ if and only if $A(W A)^{D}=(A W)^{D} B$. 
(c) $A \preceq^{D, W} B$ if and only if $A(W A)^{D}=B(W A)^{D}$ and $(A W)^{D} A=(A W)^{D} B$ if and only if $(A W)^{D} A=B(W A)^{D}$ and $A(W A)^{D}=(A W)^{D} B$.

Proof. By definition, $A \preceq^{D, W, r} B$ if and only if $(A W) A^{D, W}=(B W) A^{D, W}$. By replacing in this last equality the expressions given in (1) we get $A(W A)^{D}=B(W A)^{D}$ or $(A W)^{D} A=B(W A)^{D}$. Hence, item (a) holds. The other two items follow similarly.

We close this section emphasizing that the relations $\preceq^{D, W, r}, \preceq^{D, W, \ell}$ and $\preceq^{D, W}$ are pairwise different. It is enough to show that $A \preceq^{D, W, r} B$ does not imply $A \preceq^{D, W, \ell} B$.

Example 3.2 Let us consider the matrices

$$
A=\left[\begin{array}{llll}
2 & 0 & 0 & 0 \\
0 & 1 & 0 & 0 \\
0 & 0 & 0 & 1
\end{array}\right], \quad B=\left[\begin{array}{llll}
2 & 0 & 1 & 1 \\
0 & 1 & 0 & 0 \\
0 & 0 & 0 & 1
\end{array}\right], \quad W=\left[\begin{array}{lll}
1 & 0 & 0 \\
0 & 1 & 0 \\
0 & 0 & 1 \\
0 & 0 & 0
\end{array}\right] .
$$

By Theorem 3.2 and Theorem 3.4 it can be easily verified that $A \preceq^{D, W, r} B$ but $A \npreceq^{D, W, \ell} B$ because $(A W)^{D} A \neq(A W)^{D} B$.

\section{Some weighted partial orders}

Considering the nilpotent parts of the matrices, in [10, Definition 4.4.17] it was defined a binary relation on square matrices in order to extend the Drazin pre-order to a partial order. In this section, we define and investigate a similar relation, in this occasion on the rectangular matrices setting.

In what follows, we will denote by $(Z)_{C}$ and $(Z)_{N}$ the unique matrices in the corenilpotent decomposition of a square matrix $Z$.

Definition 4.1 Let $W \in \mathbb{C}^{n \times m}$ be a nonzero matrix and $A, B \in \mathbb{C}^{m \times n}$. It is said that:

(a) $A \preceq^{\#,-, W, r} B$ if $(A W)_{C} \leq^{\#}(B W)_{C}$ and $(A W)_{N} \leq^{-}(B W)_{N}$.

(b) $A \preceq^{\#,-, W, \ell} B$ if $(W A)_{C} \leq^{\#}(W B)_{C}$ and $(W A)_{N} \leq^{-}(W B)_{N}$.

(c) $A \preceq^{\#,-, W} B$ if $A \preceq^{\#,-, W, r} B$ and $A \preceq^{\#,-, W, \ell} B$. 
From [10, Corollary 4.4.19], we can deduce that the relations previously defined are pre-orders on $\mathbb{C}^{m \times n}$.

Theorem 4.1 Let $W \in \mathbb{C}^{n \times m}$ be a nonzero matrix and $A, B \in \mathbb{C}^{m \times n}$. The following conditions are equivalent:

(a) $A \preceq \#,-, W, r \quad B$.

(b) There exist nonsingular matrices $P, Q, A_{1}, W_{1}, B_{1}^{2}, W_{1}^{2}$, and matrices $B_{3}, A_{2}^{2}, B_{2}^{2}$, $W_{2}^{2}$ and $A_{3}^{2}$, all of them of suitable sizes, such that

$$
\begin{gathered}
A=P\left(A_{1} \oplus\left[\begin{array}{ll}
O & A_{3}^{2} \\
O & A_{2}^{2}
\end{array}\right]\right) Q^{-1}, \quad B=P\left[\begin{array}{cc}
A_{1} & B_{3} \\
O & B_{1}^{2} \oplus B_{2}^{2}
\end{array}\right] Q^{-1} \\
\text { and } \quad W=Q\left(W_{1} \oplus\left(W_{1}^{2} \oplus W_{2}^{2}\right)\right) P^{-1},
\end{gathered}
$$

where $A_{2}^{2} W_{2}^{2}, W_{2}^{2} A_{2}^{2}, B_{2}^{2} W_{2}^{2}$ and $W_{2}^{2} B_{2}^{2}$ are nilpotent matrices, $A_{3}^{2} W_{2}^{2}=O, B_{3}\left(W_{1}^{2} \oplus\right.$ $\left.W_{2}^{2}\right)=O$ and $A_{2}^{2} W_{2}^{2} \leq^{-} B_{2}^{2} W_{2}^{2}$.

Proof. (a) $\Longrightarrow$ (b) Suppose $A \preceq \#,-, W, r$, this is $(A W)_{C} \leq^{\#}(B W)_{C}$ and $(A W)_{N} \leq^{-}$ $(B W)_{N}$. From the first condition we have $A W \preceq^{d} B W$, i.e., $A \preceq^{D, W, r} B$. So, by $[9$, Theorem 2.1], there exist nonsingular matrices $P \in \mathbb{C}^{m \times m}, Q \in \mathbb{C}^{n \times n}, A_{1}, W_{1} \in \mathbb{C}^{t_{A} \times t_{A}}$, $B_{1}^{2}, W_{1}^{2} \in \mathbb{C}^{t \times t}$, and there exist matrices $A_{2} \in \mathbb{C}^{\left(m-t_{A}\right) \times\left(n-t_{A}\right)}, B_{3} \in \mathbb{C}^{t_{A} \times\left(n-t_{A}\right)}, B_{2}^{2} \in$ $\mathbb{C}^{\left(m-t_{A}-t\right) \times\left(n-t_{A}-t\right)}, W_{2}^{2} \in \mathbb{C}^{\left(n-t_{A}-t\right) \times\left(m-t_{A}-t\right)}$ satisfying

$$
\begin{gathered}
A=P\left(A_{1} \oplus A_{2}\right) Q^{-1}, \quad B=P\left[\begin{array}{cc}
A_{1} & B_{3} \\
O & B_{1}^{2} \oplus B_{2}^{2}
\end{array}\right] Q^{-1}, \\
W=Q\left(W_{1} \oplus\left(W_{1}^{2} \oplus W_{2}^{2}\right)\right) P^{-1},
\end{gathered}
$$

where $A_{2}\left(W_{1}^{2} \oplus W_{2}^{2}\right),\left(W_{1}^{2} \oplus W_{2}^{2}\right) A_{2}, B_{2}^{2} W_{2}^{2}$ and $W_{2}^{2} B_{2}^{2}$ are nilpotent matrices and $B_{3}\left(W_{1}^{2} \oplus\right.$ $\left.W_{2}^{2}\right)=O$. Hence, it is easy to see that

$$
\begin{gathered}
(A W)_{N}=P\left(O_{t_{A}} \oplus A_{2}\left(W_{1}^{2} \oplus W_{2}^{2}\right)\right) P^{-1} \quad \text { and } \\
\left.(B W)_{N}=P\left(O_{t_{A}+t} \oplus B_{2}^{2} W_{2}^{2}\right)\right) P^{-1}
\end{gathered}
$$


Let

$$
Z=\left[\begin{array}{cc}
O_{t} & O \\
O & B_{2}^{2} W_{2}^{2}
\end{array}\right] \quad \text { and } \quad A_{2}\left(W_{1}^{2} \oplus W_{2}^{2}\right)=\left[\begin{array}{cc}
M_{11} & M_{12} \\
M_{21} & M_{22}
\end{array}\right],
$$

where the partition of $A_{2}\left(W_{1}^{2} \oplus W_{2}^{2}\right)$ is according to the size of the blocks of $Z$. Since $(A W)_{N} \leq^{-}(B W)_{N}$, we have $P^{-1}(A W)_{N} P \leq^{-} P^{-1}(B W)_{N} P$, this is, $A_{2}\left(W_{1}^{2} \oplus W_{2}^{2}\right) \leq^{-} Z$. By [10, Theorem 3.3.5], $\mathcal{R}\left(A_{2}\left(W_{1}^{2} \oplus W_{2}^{2}\right)\right) \subseteq \mathcal{R}(Z)$ and $\mathcal{R}\left(\left(A_{2}\left(W_{1}^{2} \oplus W_{2}^{2}\right)\right)^{*}\right) \subseteq \mathcal{R}\left(Z^{*}\right)$, from where it is directly obtained that $M_{11}=O, M_{12}=O$ and $M_{21}=O$. Hence, $A_{2}\left(W_{1}^{2} \oplus W_{2}^{2}\right)=O \oplus M_{22}$ and it then results $M_{22} \leq^{-} B_{2}^{2} W_{2}^{2}$.

On the other hand, let us consider the following partition

$$
A_{2}=\left[\begin{array}{ll}
A_{1}^{2} & A_{3}^{2} \\
A_{4}^{2} & A_{2}^{2}
\end{array}\right],
$$

according to the blocks of $B_{1}^{2} \oplus B_{2}^{2}$. So,

$$
A_{2}^{\prime}\left(W_{1}^{2} \oplus W_{2}^{2}\right)=\left[\begin{array}{cc}
A_{1}^{2} W_{1}^{2} & A_{3}^{2} W_{2}^{2} \\
A_{4}^{2} W_{1}^{2} & A_{2}^{2} W_{2}^{2}
\end{array}\right]=\left[\begin{array}{cc}
O & O \\
O & M_{22}
\end{array}\right]
$$

and we get $A_{1}^{2}=O, A_{4}^{2}=O, A_{3}^{2} W_{2}^{2}=O$ and $M_{22}=A_{2}^{2} W_{2}^{2}$. Moreover, since $\left(W_{1}^{2} \oplus W_{2}^{2}\right) A_{2}$ is nilpotent we have that $W_{2}^{2} A_{2}^{2}$ is nilpotent.

(b) $\Longrightarrow$ (a) It is straightforward.

The following two results establish characterizations for matrices related by the preorders $\preceq \#,-, W, \ell$ and $\preceq^{\#,-, W}$, respectively.

Theorem 4.2 Let $W \in \mathbb{C}^{n \times m}$ be a nonzero matrix and $A, B \in \mathbb{C}^{m \times n}$. The following conditions are equivalent:

(a) $A \preceq \#,-, W, \ell B$.

(b) There exist nonsingular matrices $P, Q, A_{1}, W_{1}, B_{1}^{2}, W_{1}^{2}$, and matrices $B_{4}, A_{2}^{2}, B_{2}^{2}$, $W_{2}^{2}$ and $A_{4}^{2}$, all of them of suitable sizes, such that

$$
A=P\left(A_{1} \oplus\left[\begin{array}{cc}
O & O \\
A_{4}^{2} & A_{2}^{2}
\end{array}\right]\right) Q^{-1}, \quad B=P\left[\begin{array}{cc}
A_{1} & O \\
B_{4} & B_{1}^{2} \oplus B_{2}^{2}
\end{array}\right] Q^{-1}
$$




$$
\text { and } \quad W=Q\left(W_{1} \oplus\left(W_{1}^{2} \oplus W_{2}^{2}\right)\right) P^{-1},
$$

where $W_{2}^{2} A_{2}^{2}, A_{2}^{2} W_{2}^{2}, B_{2}^{2} W_{2}^{2}$ and $W_{2}^{2} B_{2}^{2}$ are nilpotent matrices, $W_{2}^{2} A_{4}^{2}=O,\left(W_{1}^{2} \oplus\right.$ $\left.W_{2}^{2}\right) B_{4}=O$ and $W_{2}^{2} A_{2}^{2} \leq^{-} W_{2}^{2} B_{2}^{2}$.

Theorem 4.3 Let $W \in \mathbb{C}^{n \times m}$ be a nonzero matrix and $A, B \in \mathbb{C}^{m \times n}$. The following conditions are equivalent:

(a) $A \preceq \#,-, W B$.

(b) There exist nonsingular matrices $P \in \mathbb{C}^{m \times m}, Q \in \mathbb{C}^{n \times n}, A_{1}, W_{1} \in \mathbb{C}^{t_{A} \times t_{A}}, B_{1}^{2}, W_{1}^{2} \in$ $\mathbb{C}^{t \times t}$, and matrices $A_{2}^{2}, B_{2}^{2} \in \mathbb{C}^{\left(m-t_{A}-t\right) \times\left(n-t_{A}-t\right)}$ and $W_{2}^{2} \in \mathbb{C}^{\left(n-t_{A}-t\right) \times\left(m-t_{A}-t\right)}$ such that

$$
\begin{gathered}
A=P\left(A_{1} \oplus\left(O_{t} \oplus A_{2}^{2}\right)\right) Q^{-1}, \quad B=P\left(A_{1} \oplus\left(B_{1}^{2} \oplus B_{2}^{2}\right)\right) Q^{-1} \\
\text { and } \quad W=Q\left(W_{1} \oplus\left(W_{1}^{2} \oplus W_{2}^{2}\right)\right) P^{-1}
\end{gathered}
$$

where $A_{2}^{2} W_{2}^{2}, W_{2}^{2} A_{2}^{2}, B_{2}^{2} W_{2}^{2}$ and $W_{2}^{2} B_{2}^{2}$ are nilpotent matrices, $A_{2}^{2} W_{2}^{2} \leq^{-} B_{2}^{2} W_{2}^{2}$ and $W_{2}^{2} A_{2}^{2} \leq^{-} W_{2}^{2} B_{2}^{2}$.

Theorem 4.4 The relations $\preceq \#,-, W, r, \preceq \#,-, W, \ell$ and $\preceq \#,-, W$ are partial orders on $\mathbb{C}^{m \times n}$ provided that $W$ has full row rank, full column rank or full rank, respectively.

It is remarkable that:

(a) $\preceq^{D, W, r}=\preceq^{d, W, r}$ if and only if Theorem 3.2 (f) also includes the condition $B_{3}\left(\left(W_{1}^{2} \oplus\right.\right.$ $\left.W_{2}^{2}\right)=O$.

(b) $\preceq^{D, W, r} \subseteq \preceq \#,-, W, r$ if and only if conditions included in Theorem 4.1 (b) and neither included in Theorem 3.2 (f) are satisfied.

We close this paper pointed out that the pre-orders defined in this section are essentially different from the ones analyzed in Sections 2 and 3. 


\section{References}

[1] A.A. Alieva, A.E. Guterman, Monotone linear transformations on matrices are invertible, Communications in Algebra, 33 (2005), 3335-3352.

[2] O.M. Baksalary, G. Trenkler, Core inverse of matrices, Linear and Multilinear Algebra, 58 (2010), 681-697.

[3] O.M. Baksalary, G. Trenkler, On a generalized core inverse, Applied Mathematics and Computation, 236 (2014), 450-457.

[4] S.L. Campbell, C.D. Meyer Jr., Generalized Inverse of Linear Transformations. Dover, New York, Second Edition, 1991.

[5] N. Castro-González, J.Y. Vélez-Cerrada, The weighted Drazin inverse of perturbed matrices with related support idempotents, Applied Mathematics and Computation, 187 (2007), 756-764.

[6] R. E. Cline, T. N. E. Greville, A Drazin inverse for rectangular matrices, Linear Algebra Appl., 29 (1980), 53-62.

[7] A. Hernández, M. Lattanzi, N. Thome, F. Urquiza, The star partial order and the eigenprojection at 0 on EP matrices, Applied Mathematics and Computation, 218, 21 (2012), $10669-10678$.

[8] A. Hernández, M. Lattanzi, N. Thome, On a partial order defined by the weighted Moore-Penrose inverse, Applied Mathematics and Computation, 219, 14 (2013), 7310 $-7318$.

[9] A. Hernández, M. Lattanzi, N. Thome, Weighted binary relations involving the Drazin inverse, Applied Mathematics and Computation, 253 (2015), 215-223.

[10] S. K. Mitra, P. Bhimasankaram, S. B. Malik, Matrix partial orders, shorted operators and applications. World Scientific Publishing Company, 2010.

[11] P. Patrício, C. Mendes Araújo, Moore-Penrose invertibility in involutory rings: the case $a a^{\dagger}=b b^{\dagger}$, Linear and Multilinear Algebra, 58, 4 (2010), 445-452. 
[12] D.S. Rakić, D.S. Djordjević, Space pre-order and minus partial order for operators on Banach spaces. Aequationes Mathematicae, 85 (2013), 429-448.

[13] M. Tošić, D.S. Cvetković-Ilić, Invertibility of a linear combination of two matrices and partial orderings, Applied Mathematics and Computation, 218, 9 (2012), 4651-4657.

[14] G. Wang, Y. Wei, S. Qiao, Generalized Inverses: Theory and Computations, Science Press, Beijing/New York, 2004.

[15] Y. Wei, Integral representation of the W-weighted Drazin inverse, Applied Mathematics and Computation, 144 (2003), 3-10. 